MATHEMATICS OF COMPUTATION

Volume 67, Number 222, April 1998, Pages 603-617

S 0025-5718(98)00922-3

\title{
ON THE NON-EXISTENCE OF $\varepsilon$-UNIFORM FINITE DIFFERENCE METHODS ON UNIFORM MESHES FOR SEMILINEAR TWO-POINT BOUNDARY VALUE PROBLEMS
}

\author{
PAUL A. FARRELL, JOHN J. H. MILLER, EUGENE O'RIORDAN, AND \\ GRIGORII I. SHISHKIN
}

\begin{abstract}
In this paper fitted finite difference methods on a uniform mesh with internodal spacing $h$, are considered for a singularly perturbed semilinear two-point boundary value problem. It is proved that a scheme of this type with a frozen fitting factor cannot converge $\varepsilon$-uniformly in the maximum norm to the solution of the differential equation as the mesh spacing $h$ goes to zero. Numerical experiments are presented which show that the same result is true for a number of schemes with variable fitting factors.
\end{abstract}

\section{INTRODUCTION}

In this paper fitted finite difference methods on a uniform mesh are considered for a singularly perturbed semilinear two-point boundary value problem. Singularly perturbed differential equations are all pervasive in applications of mathematics to problems in the sciences and engineering. Among these are the Navier-Stokes equations of fluid flow at high Reynolds number, the drift-diffusion equations of semiconductor device physics [19], [10], the Michaelis-Menten theory for enzyme reactions [16], and mathematical models of liquid crystal materials and of chemical reactions [27].

The use of classical numerical methods for solving such problems may give rise to difficulties when the singular perturbation parameter $\varepsilon$ is small. In particular, methods based on centered differences or upwinded differences on uniform meshes yield error bounds, in the maximum norm, which depend on an inverse power of $\varepsilon$. Similarly Brandt and Yavneh [1] demonstrated that anisotropic artificial viscosity in the first-order upwind finite difference scheme may result in inaccurate solutions, when $\varepsilon / h=O(1)$, where $h$ is the mesh width. Two alternative approaches may be taken to the resolution of this problem. Either additional information about the solution may be used to produce accurate efficient methods, which may involve $a$ priori modification of the mesh or operator, or an attempt may be made to produce a postiori adaptive methods or black box methods.

Received by the editor July 3, 1995 and, in revised form, February 9, 1996.

1991 Mathematics Subject Classification. Primary 34B15, 65L12; Secondary 34L30, 65L10.

Key words and phrases. Semilinear boundary value problem, singular perturbation, finite difference scheme, $\varepsilon$-uniform convergence, uniform mesh, frozen fitting factor.

Supported in part under NSF grant DMS-9627244.

The first author was supported in part by the Research Council of Kent State University.

The fourth author was supported in part by the Russian Foundation for Basic Research under Grant N 95-01-00039.

(C)1998 American Mathematical Society 
The latter approach leads to codes that are designed to handle a wider variety of problems than non-adaptive codes, usually at the expense of greater execution time. Moreover, such methods are less suitable than non-adaptive codes to implementation in a parallel environment. This is because the adaption process inherent in a posteriori methods, introduces sequentiality to the solution process, which is absent in the a priori case. The a priori approach uses physical or mathematical knowledge about the problem to enhance the solution strategy. Such methods are widespread in the literature. These include fitted finite difference methods [2], finite element methods using special elements such as exponential elements [18], and methods which use a priori refined or special meshes [12]. Examples of these include methods for convection-diffusion problems devised by the British Central Electricity Generating Board [14], fluid flow in aerodynamics [3], semiconductor device physics [20], [5], [15], [13], chemical reactions [26], and hydrologic models for the Nash cascade model of flood routing [25].

It is of theoretical and practical interest to consider numerical methods for such problems, which exhibit $\varepsilon$-uniform convergence, that is, numerical methods for which there exists an $N_{0}$, independent of $\varepsilon$, such that for all $N \geq N_{0}$, where $N$ is the number of mesh elements, the error constant and rate of convergence in the maximum norm are independent of $\varepsilon$. Thus a numerical method is said to be $\varepsilon$ uniform of order $p$ on the mesh $\Omega_{N}=\left\{x_{i}, i=0,1, \ldots, N\right\}$ if there exists an $N_{0}$ independent of $\varepsilon$ such that for all $N \geq N_{0}$

$$
\sup _{0<\varepsilon \leq 1} \max _{\Omega_{N}}\left|u(x)-u_{N}(x)\right| \leq C N^{-p},
$$

where $u$ is the solution of the differential equation, $u_{N}$ is the numerical approximation to $u, C$ and $p>0$ are independent of $\varepsilon$ and $N$.

Singularly perturbed boundary value problems for linear elliptic equations, which reduce for $\varepsilon=0$ to zero-order equations, were examined in [9], [21], [22], [23], [24]. For such problems $\varepsilon$-uniform methods consisting of exponentially fitted finite difference operators on uniform meshes were thoroughly investigated and applied successfully to ordinary differential equations in [2], [8] and to linear partial differential equations in [9], [21], [22], [23]. A sufficient condition for $\varepsilon$-uniform convergence, for linear ordinary differential equations, is that the scheme be fitted with the appropriate constant fitting factor in the region of the boundary layer. This was shown for the non-selfadjoint case in [4]. Schemes with constant exponential fitting factors (a special case of the frozen fitting factor schemes considered in this paper) for the linear self-adjoint problem were considered in [2, Chap. 10], and shown there to be $\varepsilon$-uniform.

The semilinear problem considered in this paper exhibits an exponential boundary layer, which is asymptotically similar in behavior to the layers arising in selfadjoint linear ordinary differential equations. It has been an area of speculation in the community, which considers $\varepsilon$-uniformly convergent methods, whether results of the type available widely in the literature for linear problems could also be obtained for nonlinear equations using fitted finite difference methods on uniform meshes. Previous attempts in this direction include schemes which are $\varepsilon$-uniformly convergent in weaker norms, such as the $\ell_{1}$ and $\ell_{2}$ norm (cf. Niijima [17]). The key issue in this paper is to show that, even in the case of this very simple nonlinearity, $\varepsilon$-uniform convergence cannot be achieved in the $\ell_{\infty}$ norm using fitted finite difference methods on uniform meshes. 
In this paper, it is shown that a general class of fitted finite difference methods on a uniform mesh, which includes well known exponentially fitted finite difference methods [2], are not $\varepsilon$-uniform pointwise in the maximum norm for a singularly perturbed semilinear two-point boundary value problem. To be precise, in section 2, we shall prove this result for schemes with a frozen fitting factor. The fitting factor is said to be frozen if, at points $x_{i}$ in a neighborhood of the boundary layer at $x=0$, it is determined by the quantities given at $x=0$ alone. In section 3 numerical results are given, which indicate that this result holds not only for schemes with a frozen fitting factor but also for some standard fitted schemes from the literature, the fitting factors of which are not frozen.

It should be noted however that the result does not indicate that fitted methods on non-uniform meshes cannot be $\varepsilon$-uniform. In fact, in [7], numerical methods, $\varepsilon$-uniform in the maximum norm, are constructed for a class of semilinear problems, using classical finite difference operators on special piecewise-uniform meshes. Thus $\varepsilon$-uniform methods can be constructed on special piecewise-uniform meshes even though it is not possible on uniform meshes.

\section{TheORETICAL RESUlT FOR FROZEN FITTING FACTORS}

In this section the class $\mathcal{C}$ of semilinear two-point boundary value problems on $\Omega=(0,1)$ of the form

$$
(P) \quad\left\{\begin{aligned}
\varepsilon^{2} u^{\prime \prime}(x)-c(u(x)) u(x) & =0, x \in \Omega, \\
u(0)=1, u(1) & =0
\end{aligned}\right.
$$

are considered. Here $c$ is a smooth function satisfying

$$
c(u(x)) \geq \alpha>0, x \in \Omega,
$$

and the singular perturbation parameter $\varepsilon$ satisfies $\varepsilon>0$. When $\varepsilon<<1$ the solutions of such problems exhibit boundary layers in small neighborhoods of the boundary point $x=0$. These boundary layers are the cause of significant numerical difficulties, some consequences of which are given in the theorem below.

A finite difference method is considered on a uniform mesh $\Omega_{N}=\left\{x_{i}\right\}_{1}^{N-1}$, where $x_{i}=i h, 0 \leq i \leq N$ and $N h=1$. On this mesh the standard second order central difference operator $\delta_{x}^{2}$ is used to approximate the second-order derivative, where $\delta_{x}^{2}$ is defined by:

$$
\delta_{x}^{2} w\left(x_{i}\right)=\frac{w\left(x_{i+1}\right)-2 w\left(x_{i}\right)+w\left(x_{i-1}\right)}{h^{2}},
$$

for any mesh function $w$. The discrete problem corresponding to continuous problem $(P)$ is then

$$
\left(P_{h}\right)\left\{\begin{array}{l}
\varepsilon^{2} \gamma_{i} \delta_{x}^{2} z\left(x_{i}\right)-c\left(z\left(x_{i}\right)\right) z\left(x_{i}\right)=0, x_{i} \in \Omega_{N}, \\
z(0)=1, z(1)=0,
\end{array}\right.
$$

where $\gamma_{i}$ is the fitting factor. In general, the fitting factor $\gamma_{i}$ is determined at each point $x_{i} \in \Omega_{N}$ by the quantities $\varepsilon, h, c\left(z\left(x_{i-1}\right)\right), c\left(z\left(x_{i}\right)\right)$ and $c\left(z\left(x_{i+1}\right)\right)$. The fitting factor is said to be frozen if, at points $x_{i}$ in a neighborhood of the boundary layer at $x=0$, it is determined by the quantities $\varepsilon, h, c(z(0))$ alone.

The main theoretical result of this paper states that there is no fitted central 
finite difference method $\left(P_{h}\right)$ with a frozen fitting factor on a uniform mesh, whose solutions converge $\varepsilon$-uniformly to the solution of problem $(P)$.

Theorem 2.1. Let $u$ be the solution of any problem $(P)$ in the class $\mathcal{C}$ and $z$ the solution of the corresponding discrete problem $\left(P_{h}\right)$ on the uniform mesh $\Omega_{N}$. Assume that the fitting factor $\gamma$ depends continuously on its arguments and that it is frozen so that for all $x \in[0,1 / 4), \gamma(x)=\gamma(\varepsilon, h, c(z(0)))$. Then, there is no choice of the fitting factor $\gamma$ for which the solutions of $\left(P_{h}\right)$ converge $\varepsilon$-uniformly to the solution $u$ of $(P)$, as $N \rightarrow \infty$ for all problems $(P)$ in $\mathcal{C}$.

Proof. The theorem is proved by assuming that it is false and then deriving a contradiction. Thus, it is assumed that for all problems $(P)$ in $\mathcal{C}$, there is an $\varepsilon$ uniform fitted finite difference method $\left(P_{h}\right)$ on the uniform mesh $\Omega_{N}$, with a frozen fitting factor such that $\gamma(x)=\gamma(\varepsilon, h, c(z(0)))$ for all $x \in[0,1 / 4)$, with $\gamma$ depending continuously on its arguments. That is, there exists $\mu=\mu(h)$, independent of $\varepsilon$, such that $\left|u\left(x_{i}\right)-z\left(x_{i}\right)\right| \leq \mu(h)$ where $\mu(h) \rightarrow 0$ as $h \rightarrow 0$.

Under these assumptions it will be shown that for any choice of the fitting factor the error at the point $x_{1}$, namely $u\left(x_{1}\right)-z\left(x_{1}\right)$, does not converge to zero as $N \rightarrow \infty$ for a sequence of problems in $\mathcal{C}$ for which $\varepsilon N$ is held constant. This provides the required contradiction.

It suffices to consider problems in $\mathcal{C}$ corresponding to the following two choices of the coefficient $c$,

$$
c=c_{s}(u(x))=2-s+s u(x), s=0,1 .
$$

The corresponding solutions of $(P)$ and $\left(P_{h}\right)$ are denoted by $u_{s}$ and $z_{s}$ respectively. It will be shown that either $u_{0}\left(x_{1}\right)-z_{0}\left(x_{1}\right)$ or $u_{1}\left(x_{1}\right)-z_{1}\left(x_{1}\right)$ does not converge to zero as $N \rightarrow \infty$ for the sequence of problems with $\varepsilon N=1$.

It is clear that the coefficient in (2.2) fulfills condition (2.1) for the linear problem corresponding to $s=0$. That the same is true when $0<s \leq 1$ may be verified by a standard argument using the maximum principle.

It is more convenient to work with the following auxiliary problems in the semiinfinite domain $[0, \infty)$ :

$$
\begin{gathered}
\varepsilon^{2} v_{s}^{\prime \prime}(x)-c_{s}\left(v_{s}(x)\right) v_{s}(x)=0, x \in[0, \infty), \\
v_{s}(0)=1, v_{s}(\infty)=0 .
\end{gathered}
$$

The exact solution of the linear problem corresponding to $s=0$ is $v_{0}(x)=e^{-\sqrt{2} \varepsilon^{-1} x}$. Again, using a standard maximum principle argument, it is not hard to show that for $0<s \leq 1$ the solutions $v_{s}(x)$ satisfy

$$
e^{-\sqrt{2} \varepsilon^{-1} x} \leq v_{s}(x) \leq e^{-\sqrt{2-s} \varepsilon^{-1} x}, x \in[0, \infty) .
$$

Moreover, on the interval $\bar{\Omega}=[0,1]$, the difference between the solution $u_{s}$ and the solution $v_{s}$ of the corresponding auxiliary problem decreases as $\varepsilon \rightarrow 0$ in the sense that

$$
\left|u_{s}(x)-v_{s}(x)\right| \leq \nu(\varepsilon), x \in \bar{\Omega}, s=0,1,
$$

where $\nu(\varepsilon) \rightarrow 0$ as $\varepsilon \rightarrow 0$.

Changing from $x$ to the new variable $\eta(x)=x / \varepsilon$ the auxiliary problems become

$$
\begin{gathered}
w_{s}^{\prime \prime}(\eta)-c_{s}\left(w_{s}(\eta)\right) w_{s}(\eta)=0, \eta \in[0, \infty), \\
w_{s}(0)=1, w_{s}(\infty)=0,
\end{gathered}
$$


where $w_{s}(\eta)=v_{s}(x)$. Letting $w_{s}(\eta)$ denote the solution of this problem, the function $\beta(s)$ is chosen to satisfy the difference equation

$$
\beta(s) \delta_{\eta}^{2} w_{s}(\eta)-c_{s}\left(w_{s}(0)\right) w_{s}(\eta)=0,
$$

at the point $\eta=\varepsilon^{-1} h$. Since $w_{s}(0)=1$ it follows that $c_{s}\left(w_{s}(0)\right)=c_{s}(1)=2$ for all $0 \leq s \leq 1$ and $\beta(s)$ satisfies

$$
\beta(s)\left[\delta_{\eta}^{2} w_{s}(\eta)\right]_{\eta=\ell}-2 w_{s}(\ell)=0,
$$

where $\ell=\varepsilon^{-1} h$.

To obtain an approximate expression for $\beta(s)$ as a power series in $\ell$, the function $w_{s}(\eta)$ is expanded as a power series in $\eta$

$$
w_{s}(\eta)=1+\sum_{r=1}^{\infty} k_{r}(s) \eta^{r}
$$

Then, by (2.5) and (2.6),

$$
\begin{aligned}
\beta(s) & =\frac{2\left(1+\sum_{r=1}^{\infty} k_{r}(s) \ell^{r}\right)}{\sum_{r=2}^{\infty} k_{r}(s)\left[\delta_{\eta}^{2} \eta^{r}\right]_{\eta=\ell}} \\
& =\frac{1+k_{1}(s) \ell+k_{2}(s) \ell^{2}+O\left(\ell^{3}\right)}{k_{2}(s)+3 k_{3}(s) \ell+7 k_{4}(s) \ell^{2}+O\left(\ell^{3}\right)} .
\end{aligned}
$$

Expressions for the functions $k_{r}(s)$ are now obtained by substituting the expansion of $w_{s}(\eta)$ into the differential equation satisfied by $w_{s}(\eta)$, namely

$$
w_{s}^{\prime \prime}(\eta)=\left(2-s+s w_{s}(\eta)\right) w_{s}(\eta),
$$

and so

$$
\left(\sum_{r=0}^{\infty} k_{r}(s) \eta^{r}\right)^{\prime \prime}=\left(2-s+s \sum_{r=0}^{\infty} k_{r}(s) \eta^{r}\right)\left(\sum_{r=0}^{\infty} k_{r}(s) \eta^{r}\right) .
$$

Equating coefficients of $1, \eta$ and $\eta^{2}$ on both sides and simplifying, the relations

$$
k_{2}(s)=1,6 k_{3}(s)=(2+s) k_{1}(s), 12 k_{4}(s)=2+s+s k_{1}^{2}(s)
$$

are obtained.

Writing $w_{s}(2 \ell)=e^{-\kappa(s) \ell}$, for some $\kappa(s)$, and expanding as a power series in $\ell$, we obtain

$$
w_{s}(2 \ell)=1+\sum_{r=1}^{\infty}(-1)^{r} \frac{\kappa^{r}(s) \ell^{r}}{r !} .
$$

Comparing this with the expansion (2.6) when $\eta=2 \ell$, and using $k_{2}(s)=1$ from (2.8), it follows that

$$
k_{1}(s)=-\frac{\kappa(s)}{2}+\left(\frac{\kappa^{2}(s)}{4}-2\right) \ell+O\left(\ell^{2}\right) .
$$


Using (2.8) and (2.9), the expression (2.7) for $\beta(s)$ becomes

$$
\begin{aligned}
& \beta(s)=\frac{1+\left[-\frac{\kappa(s)}{2}+\left(\frac{\kappa^{2}(s)}{4}-2\right) \ell\right] \ell+\ell^{2}+O\left(\ell^{3}\right)}{1+\frac{1}{2}(2+s)\left[-\frac{\kappa(s)}{2}+\left(\frac{\kappa^{2}(s)}{4}-2\right) \ell\right] \ell+\frac{7}{12}\left[2+s+s \frac{\kappa^{2}(s)}{4}\right] \ell^{2}+O\left(\ell^{3}\right)} \\
& =\left[1-\frac{\kappa(s) \ell}{2}+\left(-1+\frac{\kappa^{2}(s)}{4}\right) \ell^{2}+O\left(\ell^{3}\right)\right]\left[1-\frac{(2+s) \kappa(s) \ell}{4}+\right. \\
& \left.+\left(-\frac{5(2+s)}{12}+\frac{(12+13 s) \kappa^{2}(s)}{48}\right) \ell^{2}+O\left(\ell^{3}\right)\right]^{-1} \\
& =\left[1-\frac{\kappa(s) \ell}{2}+\left(-1+\frac{\kappa^{2}(s)}{4}\right) \ell^{2}+O\left(\ell^{3}\right)\right]\left[1+\frac{(2+s) \kappa(s) \ell}{4}-\right. \\
& \left.-\left(-\frac{5(2+s)}{12}+\frac{(12+13 s) \kappa^{2}(s)}{48}\right) \ell^{2}+\frac{(2+s)^{2} \kappa^{2}(s) \ell^{2}}{16}+O\left(\ell^{3}\right)\right] \\
& =1-\frac{\ell^{2}}{6}+\frac{\ell}{4}\left\{s \kappa(s)+\ell\left[\frac{5 s}{3}+\kappa^{2}(s)\left(-1-\frac{13 s}{12}+1+s+\frac{s^{2}}{4}-1-\frac{s}{2}+1\right)\right]\right\} \\
& +O\left(\ell^{3}\right) \\
& =1-\frac{\ell^{2}}{6}+\frac{\ell s}{4}\left\{\kappa(s)+\ell\left[\frac{5}{3}-\frac{7 \kappa^{2}(s)}{12}+\frac{s \kappa^{2}(s)}{4}\right]\right\}+O\left(\ell^{3}\right) \\
& =\bar{\beta}(s)+O\left(\ell^{3}\right),
\end{aligned}
$$

where

$$
\bar{\beta}(s)=1-\frac{\ell^{2}}{6}+\frac{\ell s}{4}\left\{\kappa(s)+\ell\left[\frac{5}{3}-\frac{7}{12} \kappa^{2}(s)+\frac{s \kappa^{2}(s)}{4}\right]\right\} .
$$

The following bounds for $\kappa(s)$ are obtained from $(2.3)$

$$
2 \sqrt{2-s} \leq \kappa(s) \leq 2 \sqrt{2} .
$$

From (2.10)

$$
\begin{aligned}
& \bar{\beta}(0)=1-\frac{\ell^{2}}{6}, \\
& \bar{\beta}(1)=1-\frac{\ell^{2}}{6}+\frac{\ell}{4}\left\{\kappa(1)+\ell\left[\frac{5}{3}-\frac{7}{12} \kappa^{2}(1)+\frac{\kappa^{2}(1)}{4}\right]\right\}
\end{aligned}
$$

and so

$$
\bar{\beta}(1)-\bar{\beta}(0)=\frac{\kappa(1) \ell}{4}+O\left(\ell^{2}\right) .
$$

It follows from (2.11) that $\kappa(1) \geq 2$ and thus, for all sufficiently small $\ell$,

$$
\bar{\beta}(1)-\bar{\beta}(0) \geq \frac{\ell}{4} .
$$

Putting

$$
\beta^{*}=\bar{\beta}(0)+\frac{\ell}{8},
$$


the difference equation $(2.5)$ can be rewritten as

$$
\frac{\beta^{*}}{2\left(\beta^{*}+\ell^{2}\right)}\left(w_{s}(0)+w_{s}(2 \ell)\right)=w_{s}(\ell)+\frac{\left(\beta^{*}-\beta(s)\right) \ell^{2}}{\beta(s)\left(\beta^{*}+\ell^{2}\right)} w_{s}(\ell) .
$$

Then, for all sufficiently small $\ell$ and some constant $m_{0}>0$, from (2.10), (2.13) and $(2.3)$, it follows that

$$
\frac{\beta^{*}}{2\left(\beta^{*}+\ell^{2}\right)}\left(w_{0}(0)+w_{0}(2 \ell)\right) \geq w_{0}(\ell)+m_{0} \ell^{3}
$$

and, from (2.10), (2.12), (2.13) and (2.3),

$$
\frac{\beta^{*}}{2\left(\beta^{*}+\ell^{2}\right)}\left(w_{1}(0)+w_{1}(2 \ell)\right) \leq w_{1}(\ell)-m_{0} \ell^{3} .
$$

Consider the difference scheme $\left(P_{h}\right)$, with frozen fitting factor $\tilde{\gamma}$, applied to problems $(P)$ with the coefficient $c_{s}$ that is

$$
\left(\tilde{P}_{h}\right)\left\{\begin{array}{l}
\varepsilon^{2} \tilde{\gamma} \delta_{x}^{2} z_{s}\left(x_{i}\right)-c_{s}\left(z_{s}\left(x_{i}\right)\right) z_{s}\left(x_{i}\right)=0, x_{i} \in \Omega_{N}, \\
z_{s}(0)=1, z_{s}(1)=0
\end{array}\right.
$$

At the point $x_{1}$, this can be written in the form

$$
z_{s}\left(x_{1}\right)=\frac{\tilde{\gamma}}{2 \tilde{\gamma}+\ell^{2}\left(2-s+s z_{s}\left(x_{1}\right)\right)}\left(z_{s}\left(x_{2}\right)+z_{s}(0)\right),
$$

where $\tilde{\gamma}=\tilde{\gamma}\left(\varepsilon, h, c_{s}\left(z_{s}(0)\right)=\tilde{\gamma}\left(\varepsilon, h, c_{s}(1)\right)=\tilde{\gamma}(\varepsilon, h, 2)\right.$. Note that $\tilde{\gamma}$ is independent of $s$. We first show that $\varepsilon$-uniform convergence implies $\tilde{\gamma} \geq 0$ for all sufficiently small $h$ and $\varepsilon$, satisfying $l=h / \varepsilon=$ const. From $\tilde{P}_{h}$, it is clear that $\tilde{\gamma} \geq 0$, if $z_{0}\left(x_{1}\right) \geq 0$ and $\delta_{x}^{2} z_{0}\left(x_{1}\right)>0$.

By the maximum principle, $u_{0}(x) \geq 0, x \in(0,1 / 4]$. Now, by uniform convergence,

$$
\left|u_{s}(x)-z_{s}(x)\right| \leq \mu(h),
$$

where $\mu(h) \rightarrow 0$ as $h \rightarrow 0$. Hence

$$
z_{s}\left(x_{i}\right) \geq 0, \quad x_{i} \in(0,1 / 4], \quad h \text { sufficiently small. }
$$

It remains to show $\delta_{x}^{2} z_{0}\left(x_{1}\right) \geq 0$. To do this rewrite the equations for $u_{s}$ and $z_{s}$ in scaled coordinates $\eta(x)=x / \varepsilon$ thus:

$$
\begin{gathered}
\frac{d^{2}}{d \eta^{2}} \tilde{u}_{s}(\eta)-c_{s}\left(\tilde{u}_{s}\left(\eta_{i}\right)\right) \tilde{u}_{s}(\eta)=0, \\
\tilde{u}_{s}(0)=1, \quad \tilde{u}_{s}(1 / \varepsilon)=0,
\end{gathered}
$$

and

$$
\begin{gathered}
\tilde{\gamma} \delta_{\eta}^{2} \tilde{z}_{s}\left(\eta_{i}\right)-c_{s}\left(\tilde{z}_{s}\left(\eta_{i}\right)\right) \tilde{z}_{s}\left(\eta_{i}\right)=0, \\
\tilde{z}_{s}(0)=1 \quad, \quad \tilde{z}_{s}(1 / \varepsilon)=0 .
\end{gathered}
$$

Recalling $l=h / \varepsilon$, we have

$$
\begin{aligned}
\delta_{\eta}^{2} \tilde{z}_{s}\left(\eta_{i}\right)= & \left\{\tilde{z}_{s}\left(\eta_{i}+l\right)-2 \tilde{z}_{s}\left(\eta_{i}\right)+\tilde{z}_{s}\left(\eta_{i}-l\right)\right\} / l^{2} \\
= & \left\{\tilde{z}_{s}\left(\eta_{i}+l\right)-\tilde{u}_{s}\left(\eta_{i}+l\right)\right\} / l^{2}-2\left\{\tilde{z}_{s}\left(\eta_{i}\right)-\tilde{u}_{s}\left(\eta_{i}\right)\right\} / l^{2} \\
& +\left\{\tilde{z}_{s}\left(\eta_{i}-l\right)-\tilde{u}_{s}\left(\eta_{i}-l\right)\right\} / l^{2}+\delta_{\eta}^{2} \tilde{u}_{s}\left(\eta_{i}\right) \\
\geq & -4 \mu(h) / l^{2}+\delta_{\eta}^{2} \tilde{u}_{s}\left(\eta_{i}\right) .
\end{aligned}
$$


Now

$$
\delta_{\eta}^{2} \tilde{u}_{s}\left(\eta_{i}\right)=\frac{d^{2}}{d \eta^{2}} \tilde{u}_{s}\left(\eta_{i}+\theta_{i}\right)=c_{s}\left(\tilde{u}_{s}\right) \tilde{u}_{s}\left(\eta_{i}+\theta_{i}\right) \geq \alpha \tilde{u}_{s}\left(\eta_{i}+\theta_{i}\right), \quad 0 \leq \theta_{i} \leq l .
$$

Using (2.4), we have

$$
\left|\tilde{u}_{s}(\eta)-w_{s}(\eta)\right|=\left|u_{s}(x)-v_{s}(x)\right| \leq \nu(\varepsilon),
$$

where $\nu(\varepsilon) \rightarrow 0$ as $\varepsilon \rightarrow 0$. We now restrict our attention to the case required, that is $u_{0}(x)$. We have

$$
w_{0}\left(\eta_{1}+\theta_{1}\right)=v_{0}\left(x_{1} / \varepsilon+\theta_{1}\right) \leq v_{0}(2 h / \varepsilon)=v_{0}(2 l)=e^{-2 \sqrt{2} l} .
$$

Using (2.18), we now have

$$
\tilde{u}_{0}\left(\eta_{1}+\theta_{1}\right) \geq w_{0}\left(\eta_{1}+\theta_{1}\right)-\nu(\varepsilon)=e^{-2 \sqrt{2} l}-\nu(\varepsilon) .
$$

Thus, for $\varepsilon$ sufficiently small,

$$
\tilde{u}_{0}\left(\eta_{1}+\theta_{1}\right) \geq e^{-4 l}>0 .
$$

Hence, from (2.16), (2.17) and (2.19), since $l$ is a constant, we have

$$
\delta_{\eta}^{2} \tilde{z}_{0}\left(\eta_{1}\right) \geq-4 \mu(h) / l^{2}+e^{-4 l}>0
$$

for $h$ sufficiently small, and the required result, $\tilde{\gamma} \geq 0$ follows.

The argument is now divided into the two possible cases $\tilde{\gamma} \geq \beta^{*}$ and $\tilde{\gamma} \leq \beta^{*}$.

Suppose first that $\tilde{\gamma} \geq \beta^{*}$ and consider problems corresponding to $s=0$. Then, for all sufficiently small $\ell$, using the assumption of $\varepsilon$-uniform convergence, (2.4) and (2.14) we obtain

$$
\begin{aligned}
z_{0}\left(x_{1}\right) & =\frac{\tilde{\gamma}}{2\left(\tilde{\gamma}+\ell^{2}\right)}\left(z_{0}\left(x_{2}\right)+z_{0}(0)\right) \\
& \geq \frac{\tilde{\gamma}}{2\left(\tilde{\gamma}+\ell^{2}\right)}\left(u_{0}\left(x_{2}\right)+u_{0}(0)-\mu(h)\right) \\
& \geq \frac{\tilde{\gamma}}{2\left(\tilde{\gamma}+\ell^{2}\right)}\left(v_{0}\left(x_{2}\right)+v_{0}(0)-\nu(\varepsilon)-\mu(h)\right) \\
& =\frac{\tilde{\gamma}}{2\left(\tilde{\gamma}+\ell^{2}\right)}\left(w_{0}\left(x_{2}\right)+w_{0}(0)-\nu(\varepsilon)-\mu(h)\right) \\
& \geq \frac{\tilde{\gamma}}{2\left(\tilde{\gamma}+\ell^{2}\right)}\left[\frac{2\left(\beta^{*}+\ell^{2}\right)}{\beta^{*}}\left(w_{0}(\ell)+m_{0} \ell^{3}\right)-\nu(\varepsilon)-\mu(h)\right] \\
& \geq w_{0}(\ell)+m_{0} \ell^{3}-\nu(\varepsilon)-\mu(h) \\
& \geq u_{0}\left(x_{1}\right)+m_{0} \ell^{3}-\nu(\varepsilon)-\mu(h),
\end{aligned}
$$

since $\tilde{\gamma} \geq \beta^{*}$ implies that $\frac{\tilde{\gamma}\left(\beta^{*}+\ell^{2}\right)}{\left(\tilde{\gamma}+\ell^{2}\right) \beta^{*}} \geq 1$. Fixing $\ell$ sufficiently small, and considering the sequence of problems corresponding to $\varepsilon=\frac{h}{\ell}=\frac{1}{N \ell}$, it follows that

$$
z_{0}\left(x_{1}\right)-u_{0}\left(x_{1}\right) \geq \frac{1}{2} m_{0} \ell^{3},
$$


for all sufficiently small $h$, which contradicts the assumption of $\varepsilon$-uniform convergence of the method for these problems.

On the other hand if $\tilde{\gamma} \leq \beta^{*}$, using the assumption of $\varepsilon$-uniform convergence, (2.4) and (2.15), a similar argument for problems corresponding to $s=1$ gives for all sufficiently small $\ell$ that

$$
\begin{aligned}
z_{1}\left(x_{1}\right) & =\frac{\tilde{\gamma}}{2 \tilde{\gamma}+\ell^{2}\left(1+z_{1}\left(x_{1}\right)\right)}\left(z_{1}\left(x_{2}\right)+z_{1}(0)\right) \\
& \leq \frac{\tilde{\gamma}}{2 \tilde{\gamma}+\ell^{2}\left(1+z_{1}\left(x_{1}\right)\right)}\left[\frac{2\left(\beta^{*}+\ell^{2}\right)}{\beta^{*}}\left(w_{1}(\ell)-m_{0} \ell^{3}\right)+\nu(\varepsilon)+\mu(h)\right] \\
& \leq u_{1}(\ell)-m_{0} \ell^{3}+\nu(\varepsilon)+\mu(h),
\end{aligned}
$$

since $\tilde{\gamma} \leq \beta^{*}$ implies that $\frac{\tilde{\gamma}\left(\beta^{*}+\ell^{2}\right)}{\left(\tilde{\gamma}+\ell^{2}\right) \beta^{*}} \leq 1$.

Again, fixing $\ell$ sufficiently small, and considering the sequence of problems corresponding to $\varepsilon=\frac{h}{\ell}=\frac{1}{N \ell}$, it follows that

$$
u_{1}\left(x_{1}\right)-z_{1}\left(x_{1}\right) \geq m_{0} \ell^{3}-\nu(\varepsilon)-\mu(h) \geq \frac{1}{2} m_{0} \ell^{3},
$$

for all sufficiently small $h$, which contradicts the assumption of $\varepsilon$-uniform convergence.

Thus it has been shown that the assumption of $\varepsilon$-uniform convergence leads to a contradiction in all cases, which completes the proof of the theorem.

\section{Numerical Results}

We shall now examine numerically a number of fitted schemes on uniform meshes for the continuous problem $(P)$ and related problems with two boundary layers. We shall first consider schemes of the form

$$
\left(P_{h}\right)\left\{\begin{array}{l}
\varepsilon \gamma_{i} \delta_{x}^{2} z_{N}\left(x_{i}\right)-c\left(z_{N}\left(x_{i}\right)\right) z_{N}\left(x_{i}\right)=0, x_{i} \in \Omega_{N}, \\
z_{N}(0)=1, z_{N}(1)=0,
\end{array}\right.
$$

where $\gamma_{i} \equiv \gamma\left(\varepsilon, h, c\left(z_{N}(0)\right)\right)$ is the frozen fitting factor. Note that, in order to preserve consistency with schemes given in the literature, this and subsequent problems have an $\varepsilon$ as coefficient of the second derivative, rather than the $\varepsilon^{2}$ considered previously.

The nonlinear finite difference method $\left(P_{h}\right)$ is linearized using a continuation method of the form :

$$
\begin{gathered}
L_{t}^{h} u_{N} \equiv \varepsilon \gamma_{i} \delta_{x}^{2} u_{N}\left(x, t_{j}\right)-c\left(u_{N}\left(x, t_{j-1}\right)\right) u_{N}\left(x, t_{j}\right)-D_{t}^{-} u_{N}\left(x, t_{j}\right)=0, j=1, \ldots K, \\
u_{N}\left(0, t_{j}\right)=u(0), u_{N}\left(1, t_{j}\right)=u(1) \text { for all } j, \\
u_{N}(x, 0)=u_{\text {init }}(x),
\end{gathered}
$$

where $\gamma_{i} \equiv \gamma\left(\varepsilon, h, c\left(u_{N}\left(0, t_{j-1}\right)\right)\right) \equiv \gamma(\varepsilon, h, c(u(0)))$ is the frozen fitting factor. Various starting values $u_{i n i t}(x)$ are chosen. The number of iterations $K$ and the choice of uniform time step $h_{t}=t_{j}-t_{j-1}$ are discussed below. With the definition

$$
e(j) \equiv \max _{1 \leq i \leq N}\left|u_{N}\left(x_{i}, t_{j}\right)-u_{N}\left(x_{i}, t_{j-1}\right)\right| / h_{t}, \text { for } j=1,2, \ldots, K,
$$


the time step $h_{t}$ is chosen sufficiently small so that

$$
e(j) \leq e(j-1), \text { for } 1<j \leq K,
$$

and the number of iterations $K$ is chosen such that

$$
e(K) \leq \mathrm{TOL},
$$

where TOL is some prescribed small tolerance.

The numerical solution is obtained as follows:

Start with $h_{t}=0.0625$. If, at some value of $j,(3.1)$ is not satisfied, then halve the time step until (3.1) is satisfied. Continue the iterations until either (3.2) is satisfied or until $K=90$. If (3.2) is not satisfied, then repeat the entire process starting with $h_{t}=0.03125$.

The resulting values of $u_{N}(x, K)$ are taken as approximations to the solution of the continuous problem.

The problem is solved on a sequence of meshes, with $N=8,16,32,64,128$, $256,512,1024$ and for $\varepsilon=2^{-n}, n=1,2, \ldots j_{r e d}$, where $j_{r e d}$ is chosen so that $\varepsilon$ is a value at which the rate of convergence stabilizes, which normally occurs when, to machine accuracy, we are solving the reduced problem.

The errors $\left|u_{N}\left(x_{i}, K\right)-u\left(x_{i}\right)\right|$ are approximated on each mesh for successive values of $\varepsilon$ by $e_{\varepsilon, N}(i)=\left|u_{N}\left(x_{i}, K\right)-u^{I}\left(x_{i}, K\right)\right|$, where $u^{I}(x, K)$ is defined by linear interpolation on each subinterval $\left[y_{j-1}, y_{j}\right]$ by

$$
u^{I}(x, K)=u^{*}\left(y_{j-1}, K\right)+\left(u^{*}\left(y_{j}, K\right)-u^{*}\left(y_{j-1}, K\right)\right) \frac{x-y_{j-1}}{y_{j}-y_{j-1}}, 1 \leq j \leq 1024
$$

where the nodal values $\left\{u^{*}\left(y_{j}, K\right)\right\}_{j=0}^{1024}$ are obtained from the solution of the finite difference method $L_{t}^{h}$ with $N=1024$. For each $\varepsilon$ and each $N$ the maximum nodal error is approximated by

$$
E_{\varepsilon, N}=\max _{i} e_{\varepsilon, N}(i)
$$

For each $N$, the $\varepsilon$-uniform maximum nodal error is approximated by

$$
E_{N}=\max _{\varepsilon} E_{\varepsilon, N}
$$

A numerical method for solving $(P)$ is $\varepsilon$-uniform of order $p$ on the mesh $\Omega_{N}=$ $\left\{x_{i}, i=0,1, \ldots, N\right\}$ if

$$
\sup _{0<\varepsilon \leq 1} \max _{\Omega_{N}}\left|u(x)-u_{N}(x, K)\right| \leq C N^{-p}
$$

where $u$ is the solution of $(P), u_{N}$ is the numerical approximation to $u, C$ and $p>0$ are independent of $\varepsilon$ and $N$. An approximation to $p$, the $\varepsilon$-uniform rate of convergence, was determined using a variation of the double mesh method described in [6]. This involves calculating the double mesh error

$$
D_{\varepsilon, N}=\max _{\Omega_{N}}\left|u_{N}\left(x_{i}, K\right)-u_{2 N}^{I}\left(x_{i}, K\right)\right|
$$

which is the difference between the values of the solution on a mesh of $N$ points and the interpolated value for the solution, at the same point, on a mesh of $2 N$ 
points. For each value of $N$ the quantities

$$
D_{N}=\max _{\varepsilon} D_{\varepsilon, N}, \quad p_{N}=\log _{2}\left(\frac{D_{N}}{D_{2 N}}\right),
$$

are computed. The values of $p_{N}$ are the approximations to $p$.

We now present numerical results, first for a problem of the form $(P)$, and secondly for a generalization of that problem. All calculations were carried out in double-precision FORTRAN 77 on an Hewlett-Packard/Apollo 730. The first scheme we consider is the unfitted central difference scheme, where $\gamma_{i} \equiv 1$, and the second is a modification to the single layer case of the constant fitting factor version of the scheme of Miller [11] proposed in [2, Ch. 10, p. 156]. This gives a frozen fitting factor method for the problem

$$
\varepsilon u^{\prime \prime}(x)-c(u(x)) u(x)=0, x \in \Omega
$$

with boundary layer at $x=0$. In this case the frozen fitting factor is given by:

$$
\gamma_{i}=\frac{c(u(0)) h^{2}}{4 \varepsilon} \sinh ^{-2} \frac{\sqrt{c(u(0))} h}{2 \sqrt{\varepsilon}} .
$$

Table 1 gives uniform errors and rates of uniform convergence for the centered difference method for the problem

$$
\begin{gathered}
\varepsilon \frac{d^{2}}{d x^{2}} u(x)-u-u^{2}=0, x \in(0,1), \\
u(0)=1, \quad u(1)=0 .
\end{gathered}
$$

TABLE 1. Maximum errors $E_{N}$ and rate of convergence $p_{N}$ for the Centered Difference scheme

\begin{tabular}{|l|r|r|r|r|r|r||}
\hline \multicolumn{7}{||c||}{ Boundary Conditions: $u(0)=1.0, u(1)=0.0$} \\
Initial Guess : & \multicolumn{4}{|c||}{$u_{\text {init }}=u(x)=u(0)+(u(1)-u(0)) x$} \\
\hline$N$ & 8 & 16 & 32 & 64 & 128 & 256 \\
\hline$E_{N}$ & .048165 & .048145 & .048089 & .047872 & .047021 & .043776 \\
\hline$p_{N}$ & .00 & .00 & .00 & .00 & .00 & .00 \\
\hline
\end{tabular}

Tables 2 and 3 give errors and rates of uniform convergence for the scheme with frozen fitting factor given by (3.3).

We now show numerically that the result of Theorem 2.1 also holds for fitted methods with frozen and non-frozen fitting factors for the problem

$$
\left\{\begin{array}{c}
\varepsilon u^{\prime \prime}(x)-c(u(x)) u(x)=0, x \in \Omega, \\
u(0)=A, u(1)=B,
\end{array}\right.
$$

where $c$ satisfies (2.1). This has, in general, boundary layers at both $x=0$ and $x=1$. The nature of these boundary layers and the behavior of the derivatives of $u$ in the neighborhood of $x=0$ and $x=1$ is similar to that of the layer in $(P)$. 
TABle 2. Errors $E_{\varepsilon, N}$ and $E_{N}$ for Frozen Fitting Factor scheme (3.3)

\begin{tabular}{|c|c|c|c|c|c|c|c|}
\hline \multicolumn{8}{|c|}{ Boundary Conditions: $u(0)=1.0, u(1)=0.0$} \\
\hline \multicolumn{8}{|c|}{ Initial Guess : $\quad u_{\text {init }}=u(x)=u(0)+(u(1)-u(0$} \\
\hline \multirow[b]{2}{*}{$\epsilon$} & \multicolumn{7}{|c|}{ Number of Mesh Points $N$} \\
\hline & 8 & 16 & 32 & 64 & 128 & 256 & 512 \\
\hline $1 / 2$ & .000468 & .000120 & .000030 & .000008 & .000002 & .000000 & 000000 \\
\hline $1 / 4$ & 000819 & .000212 & .000054 & .000013 & .000003 & .000001 & 00000 \\
\hline $1 / 8$ & 001460 & .000391 & .000101 & .000025 & .000006 & 000001 & 0000 \\
\hline $1 / 16$ & 002606 & .000743 & .000 & .000049 & .000012 & .0000 & 000001 \\
\hline & .003724 & .001429 & .000 & .000098 & .000024 & & \\
\hline $1 / 64$ & .003293 & .002592 & .0007 & .000194 & .000049 & .000012 & 002 \\
\hline & .0035 & .0037 & & & .000 & & 005 \\
\hline & .007963 & .003289 & & .000 & .000192 & & \\
\hline & .0112 & .0035 & & & & & \\
\hline 1/ 1024 & & .0079 & & & & & \\
\hline & & & & & & & \\
\hline & .00019 & .006864 & & .003261 & .002544 & .000 & 146 \\
\hline & & & & & .003637 & & \\
\hline & & & & & & & 548 \\
\hline & & & & & & & \\
\hline & .000000 & .000000 & & 03 & .007 & & 852 \\
\hline $1 / 131072$ & .000000 & .000000 & .0000 & .001801 & 011111 & . 003362 & .002516 \\
\hline & & & & & & & .001833 \\
\hline & & & & & & & \\
\hline $1 / 1048576$ & .000000 & .000000 & & .000000 & .000151 & & .006751 \\
\hline $1 / 2097152$ & .000000 & .000000 & & .000000 & .000003 & .001255 & .007748 \\
\hline & & & & & & & \\
\hline & & & & & & & .000503 \\
\hline & .000000 & .000000 & & & & & .000021 \\
\hline 1/ 33554432 & .000000 & .000000 & .000000 & .000000 & .000000 & .000000 & .000000 \\
\hline$E_{N}$ & .011277 & .011277 & .011269 & .011239 & .011111 & .010508 & .007748 \\
\hline
\end{tabular}

TABLE 3. Maximum errors $E_{N}$ and rate of convergence $p_{N}$ for Frozen Fitting Factor scheme (3.3)

\begin{tabular}{|l|r|r|r|r|r|r||}
\hline \multicolumn{7}{||c||}{ Boundary Conditions: $u(0)=1.0, u(1)=0.0$} \\
Initial Guess : & \multicolumn{4}{|c||}{$u_{\text {init }}=u(x)=u(0)+(u(1)-u(0)) x$} \\
\hline$N$ & 8 & 16 & 32 & 64 & 128 & 256 \\
\hline$E_{N}$ & .011277 & .011277 & .011269 & .011239 & .011111 & .010508 \\
\hline$p_{N}$ & .00 & .00 & .00 & .00 & .00 & .00 \\
\hline
\end{tabular}

We present numerical results for the analog of (3.4) above, that is

$$
\begin{gathered}
\varepsilon \frac{d^{2}}{d x^{2}} u(x)-u-u^{2}=0, x \in(0,1), \\
u(0)=A, \quad u(1)=B .
\end{gathered}
$$

We first consider the method proposed in [2, Ch. 10, p. 159]. This gives a piecewise constant frozen fitting factor method for the problem (P2). The fitting factor is 
given by:

$$
\begin{array}{ll}
\gamma_{i}=\frac{c(u(0)) h^{2}}{4 \varepsilon} \sinh ^{-2} \frac{\sqrt{c(u(0))} h}{2 \sqrt{\varepsilon}}, \quad 0 \leq x_{i} \leq 1 / 2, \\
\gamma_{i}=\frac{c(u(1)) h^{2}}{4 \varepsilon} \sinh ^{-2} \frac{\sqrt{c(u(1))} h}{2 \sqrt{\varepsilon}}, & 1 / 2<x_{i} \leq 1 .
\end{array}
$$

Table 4 gives uniform convergence rates for this scheme.

TABLE 4. Maximum errors $E_{N}$ and rate of convergence $p_{N}$ for Frozen Fitting Factor scheme (3.6)

\begin{tabular}{|l|r|r|r|r|r|r||}
\hline \multicolumn{6}{||c||}{ Boundary Conditions: $u(0)=0.5, u(1)=0.7$} \\
Initial Guess : & \multicolumn{3}{|c||}{$u_{\text {init }}=u(x)=u(0)+(u(1)-u(0)) x$} \\
\hline$N$ & 8 & 16 & 32 & 64 & 128 & 256 \\
\hline$E_{N}$ & .006028 & .006027 & .006024 & .006010 & .005952 & .005669 \\
\hline$p_{N}$ & .00 & .00 & .00 & .00 & .00 & .00 \\
\hline
\end{tabular}

Table 5 gives rates for the scheme of Miller [11] discussed in [2, Ch. 6] which has the following (non-frozen) fitting factor:

$$
\gamma_{i}=\frac{c\left(u\left(x_{i}\right)\right) h^{2}}{4 \varepsilon} \sinh ^{-2} \frac{\sqrt{c\left(u\left(x_{i}\right)\right)} h}{2 \sqrt{\varepsilon}} .
$$

TABLE 5. Maximum errors $E_{N}$ and rate of convergence $p_{N}$ for the scheme of Miller (3.7)

\begin{tabular}{|l|r|r|r|r|r|r||}
\hline \multicolumn{8}{||c||}{ Boundary Conditions: $u(0)=0.5, u(1)=0.7$} \\
Initial Guess : & \multicolumn{4}{|c||}{$u_{\text {init }}=u(x)=u(0)+(u(1)-u(0)) x$} \\
\hline$N$ & 8 & 16 & 32 & 64 & 128 & 256 \\
\hline$E_{N}$ & .007895 & .007891 & .007882 & .007846 & .007705 & .007163 \\
\hline$p_{N}$ & .00 & .00 & .00 & .00 & .00 & .00 \\
\hline
\end{tabular}

As can be seen from these tables, none of the standard fitted schemes from the literature, on uniform meshes, are uniformly $\varepsilon$-convergent for the test problems. As remarked in the introduction, numerical methods, $\varepsilon$-uniform in the maximum norm, were constructed in [7] for a class of semilinear problems, which includes the class of problems considered here. These use classical finite difference operators on special piecewise-uniform meshes condensed or refined in the boundary layers. Thus $\varepsilon$-uniform methods can be constructed on special piecewise uniform meshes although it is not possible on uniform meshes.

\section{REFERENCES}

1. A. Brandt, I. Yavneh, Inadequacy of First-order Upwind Difference Schemes for some Recirculating Flows, J. Comput. Phys., 93, (1991) pp. 128-143. MR 91m:76075

2. E.P. Doolan, J.J.H. Miller, W.H.A. Schilders, Uniform Numerical Methods for Problems with Initial and Boundary Layers, Boole Press, Dublin, Ireland, 1980. MR 82h:65053 
3. T.M. El-Mistikawy, M.J. Werle, Numerical Method for Boundary Layers with Blowing - the Exponential Box Scheme, AIAA J., 16 (1978), pp. 749-751.

4. P.A. Farrell, Sufficient conditions for uniform convergence of a class of difference schemes for a singularly perturbed problem, IMA J. Numer. Anal., 7(4), (1987), pp 459-472. MR 90h:65130

5. P.A. Farrell, E.C. Gartland, Jr., On the Scharfetter-Gummel Discretization for DriftDiffusion Continuity Equations, in "Computational Methods for Boundary and Interior Layers in Several Dimensions", J.J.H. Miller, ed., pp. 51-79, Boole Press, Dublin, Ireland, (1991). MR 92k:65153

6. P.A. Farrell, A. Hegarty, On the determination of the order of uniform convergence, in Proc. of $13^{\text {th }}$ IMACS World Congress, Dublin, Ireland, 1991, pp. 501-502.

7. P.A. Farrell, J.J.H. Miller, E. O'Riordan, G.I. Shishkin, A uniformly convergent finite difference scheme for a singularly perturbed semilinear equation, SIAM J. Numer. Anal., 33, no. 3, (1996), pp. 1135-1149. MR 97b:65086

8. A.M. Il'in, Difference scheme for a differential equation with a small parameter affecting the highest derivative, Mat. Zametki, 6 (1969), pp. 237-248. MR 41:4823

9. V.D. Liseikin, On the numerical solution of second order equations with a small parameter affecting the highest derivatives, Chisl. Metody Mechaniki Splosh. Sredy, Novosibirsk, 14, n. 3 (1983), pp. 98-108. MR 86a:65075

10. P.A. Markowich, C.A. Ringhofer, S. Selberherr, M. Lentini, A singular perturbation approach for the analysis of the fundamental semiconductor equations, IEEE Trans. Electron Devices, 30, n. 9 (1983), pp. 1165-1180.

11. J.J.H. Miller, On the convergence, uniformly in $\varepsilon$, of difference schemes for a two-point boundary singular perturbation problem, in Numerical analysis of singular perturbation problems, P.W. Hemker, J.J.H. Miller, eds., Academic Press (1979), pp. 467-474. MR 81f:65061

12. J.J.H. Miller, E.O'Riordan, G.I.Shishkin, Fitted Numerical Methods for Singular Perturbation Problems, World Scientific, Singapore, 1996. CMP 97:10

13. J.J.H. Miller, W. Song, A Tetrahedral Mixed Finite Element Method for the Stationary Semiconductor Continuity Equations SIAM J. Numer. Anal. , 31 n. 1 (1994), pp. 196-216.

14. K.W.Morton, Numerical Solution of Convection Diffusion Problems, Chapman and Hall, London, 1996.

15. J.J.H. Miller ed., Applications of Advanced Computational Methods for Boundary and Interior Layers, Boole Press, Dublin, Ireland, (1993) MR 94e:65006

16. J.D. Murray, Lectures on Nonlinear Differential Equation Models in Biology, Clarendon Press, Oxford, 1977.

17. Koichi Niijima, An error analysis for a difference scheme of exponential type applied to a nonlinear singular perturbation problem without turning points, J. Comput. Appl. Math., 15, no. 1, (1986) pp. 93-101. MR 87h:65129

18. H.-G. Roos, M. Stynes, L. Tobiska, Numerical Methods for Singularly Perturbed Differential Equations. - Convection-Diffusion and Flow Problems, Springer-Verlag, New-York, 1996.

19. W.V. van Roosbroeck, Theory of flows of electrons and holes in germanium and other semiconductors, Bell Syst. Tech. J., 29 (1950), pp. 560-607.

20. D.L. Scharfetter, H.K. Gummel, Large-signal analysis of a silicon Read diode oscillator, IEEE Trans. Electron Devices, 16, n. 1 (1969), pp. 64-77.

21. G.I. Shishkin, Approximation of solutions of singularly perturbed boundary-value problems with a corner boundary layer, Comput. Maths. Math. Phys., 27, n. 5 (1987), pp. 54-63. (J. Vychisl. Mat. i Mat. Fiz., 27, n. 9 (1987), pp. 1360-1372.) MR 89a:65149

22. G.I. Shishkin, Grid approximation of boundary value problems with regular boundary layer, Part 1, Part 2, Preprint INCA, 1990.

23. G.I. Shishkin, Difference scheme for solving elliptic equations with small parameters affecting the derivatives, Banach Centre Publ., Warsaw, 3 (1978), pp. 89-92. MR 80d:65111

24. G.I. Shishkin, Grid approximation of singularly perturbed boundary value problems with a regular boundary layer, Sov. J. Numer. Anal. Math. Modelling, 4, n. 5 (1989), pp. 397-417. MR 91b:65138

25. Szollosi-Nagy, The Discretization of the Continuous Linear Cascade by Means of State Space Analysis J. Hydrol., 58, (1982) pp. 223-236. 
26. R. Vulanović, P.A. Farrell, P.Lin, Numerical Solution of Non-linear Singular Perturbation Problems Modeling Chemical Reactions, in "Applications of Advanced Computational Methods for Boundary and Interior Layers", J.J.H. Miller, ed., Boole Press, Dublin, Ireland, pp. 192-213 (1993) MR 95a:65119

27. V. W. Weekman, Jr., R. L. Gorring, Influence of volume change on gas-phase reactions in porous catalysts, J. Catalysis 4 (1965), 260-270.

Department of Mathematics and Computer Science, Kent State University, Kent, Оніо 44242

E-mail address: farrell@mcs.kent.edu

Department of Mathematics, Trinity College, Dublin 2, Ireland

E-mail address: jmiller@tcd.ie

School of Mathematical Sciences, Dublin City University, Glasnevin, Dublin 9, IreLAND

E-mail address: oriordae@ccmail.dcu.ie

Institute of Mathematics and Mechanics, Russian Academy of Sciences, EkaterinBURG, RUSSIA

E-mail address: grigorii@shishkin.ural.ru 